\title{
Low intense physical exercise in normobaric hypoxia leads to more weight loss in obese people than low intense physical exercise in normobaric sham hypoxia
}

\author{
Nikolaus C. Netzer • Roland Chytra • Thomas Küpper
}

Published online: 5 December 2007

(C) Springer-Verlag 2007

\begin{abstract}
Training in mild to moderate hypoxia (14-17\% $\mathrm{O}_{2}$ in breathing air) and extended resting in moderate hypoxia $\left(9-13 \% \mathrm{O}_{2}\right)$ have been shown to have effects in animals and humans on lipid and glucose metabolism, appetite loss, and, in part, on body weight. The causality for these effects is not yet known in detail, and the available data in humans from high-altitude and low-pressure chamber studies are scarce. New technical developments by German companies in the production of artificial climates with normobaric hypoxic conditions in larger rooms at reasonable energy costs allow now to perform hypoxia weight loss studies in obese humans with stable experimental conditions and protocols with a sham hypoxia control. Thirty-two obese people were recruited for a mild intense training study in normobaric hypoxia $\left(15\right.$ vol. $\left.\% \mathrm{O}_{2}\right)$ and normoxia/sham hypoxia $\left(20.1\right.$ vol.\% $\left.\mathrm{O}_{2}\right)$. Twenty of these [mean age 47.6 years, mean body mass index (BMI) $33.1,16 \mathrm{~m}, 4 \mathrm{f}$ ) were willing to follow up on an 8-week, three times per week, 90-min low intense physical exercise in their individual fat burning mode, which has been determined by an exercise testing with spiro-ergometry upfront. The subjects were evenly randomized into a hypoxia and sham hypoxia group. The difference of the two groups in weight loss and changes in $\mathrm{HBa1C}$ values were analyzed before and after the training period. No
\end{abstract}

\footnotetext{
N. C. Netzer $(\bowtie) \cdot$ R. Chytra $\cdot$ T. Küpper

Hermann Buhl Institute for Hypoxia and Sleep Medicine

Research, Paracelsus Medical University Salzburg,

Kurmittelhaus der Moderne, Salzburgerstrasse 7,

83435 Bad Reichenhall, Germany

e-mail: nikinetzer@yahoo.com

T. Küpper

Institute and Outpatient Clinic of Occupational and Social

Medicine, Aachen Technical University,

Aachen, Germany
}

nutritional diet was applied. Subjects in the hypoxia group in mean lost significantly more weight than in the sham hypoxia group $(\Delta 1.14 \mathrm{~kg}$ vs $\Delta 0.03 \mathrm{~kg} ; p=0.026)$. This resulted in a tendency to reduce the BMI more in the hypoxia group $(p=0.326)$. In the mean, there was no HbA1C exceeding normal values (mean 5.67 and 5.47\%), and the $\mathrm{HbA1C}$ stayed basically unchanged after the 8week training. Mild physical exercise three times per week for $90 \mathrm{~min}$ in normobaric hypoxia for 8 weeks led to significantly greater weight loss in obese persons than the exercise in sham hypoxia in this, to our knowledge, first sham hypoxia controlled study.

Keywords Normobaric hypoxia · Weight loss .

Physical exercise

\section{Introduction}

High altitude exposure which cause hypoxaemia has various serious effects on the human body. Those effects are studied by physiologists since the nineteenth century, and science has shed light on a lot of physiological and pathophysiological body reactions [1].

Weight loss in high altitude under hypoxic climate conditions is one of those effects in which the causes remain still unclear, and it is not known if it is dose/altitude-dependent and affects everybody or only certain individuals [3-5].

The detection of the hypoxia-induced factor-alpha 1 (HIF$\alpha 1)$ and the chain reaction on mediators that this factor starts have prompted some speculations and investigations on various nutritional signals. One of the nutritional signals that seem to be triggered by HIF is the peptide hormone leptin [69]. This could lead to the direction that the human lipid 
Fig. 1 The $90 \mathrm{~m}^{2}$ hypoxia training room with exercise devices

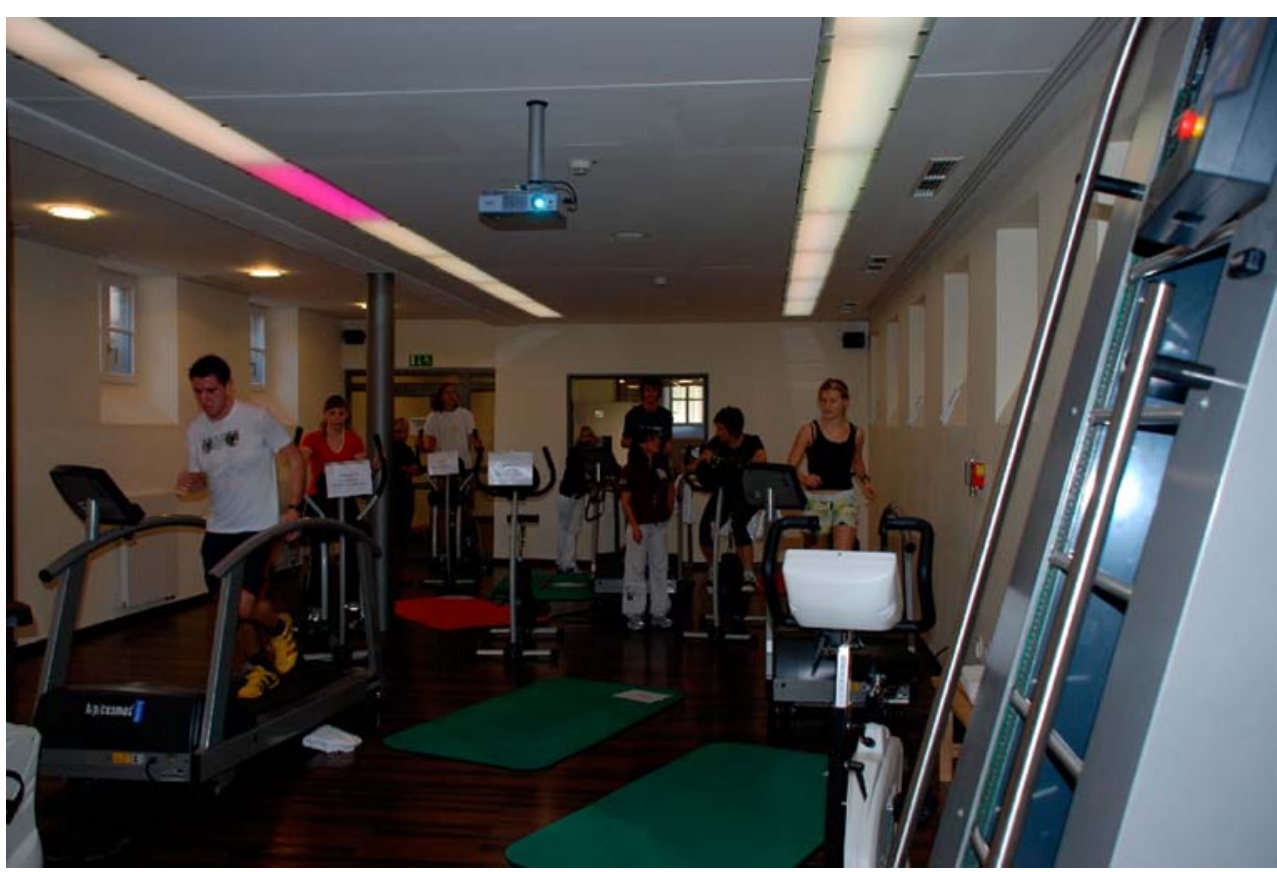

metabolism is affected by hypoxia and that hypoxia causes signals to reduce food intake and also changes in fat mobilization and fat uptake to the body [10].

Because the societies in first and second world countries struggle with an increased body weight, pathological fat levels, and the increasing number of individuals with metabolic syndrome in their populations, the urge for various inexpensive treatment forms including a lifestyle change has risen. The idea is coming up whether training or stay at moderate altitude or simulated altitude could enhance weight loss and improvement of fat and sugar metabolism because of the known effects of hypoxia on weight loss. Several open trial field studies with low

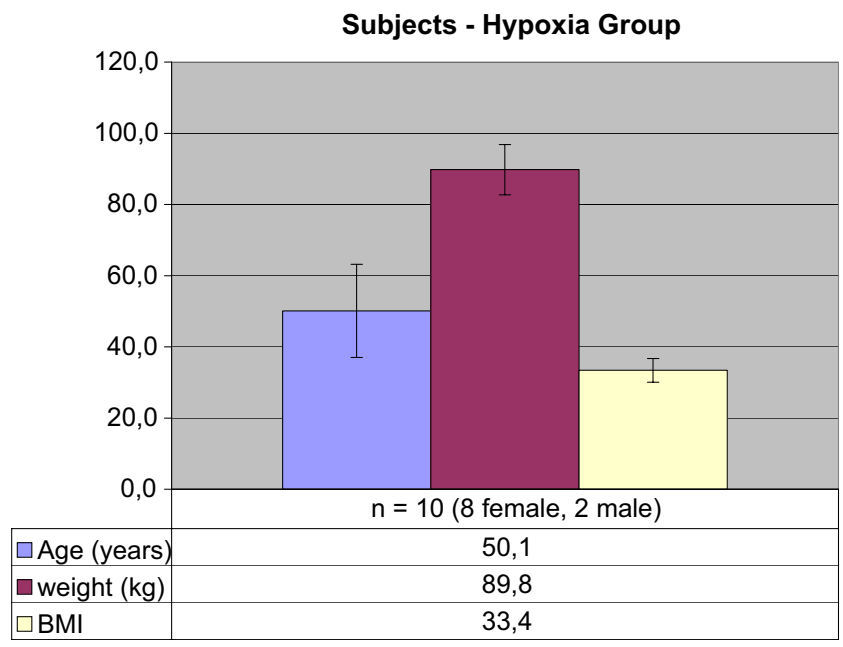

Fig. 2 Mean age (SD), weight, and body mass index (BMI) of the subjects who finished the trial in the hypoxia group numbers of individuals have been carried out so far, but they do not have controls [11-13]. In the AMAS 2000 study, patients with metabolic syndrome have trained and stayed for 3 weeks at $1,700 \mathrm{~m}$ altitude or within a control group at sea level. In this randomized controlled study, significant improvements in fat metabolism and regarding weight loss have been found for the patients at altitude compared to patients at sea level [14].

The construction design of our facility with normobaric hypoxic climate rooms allows pretending hypoxic conditions without actually running the rooms in hypoxic climate (sham hypoxia) and to perform a single blind randomized controlled weight loss study in simulated altitude.

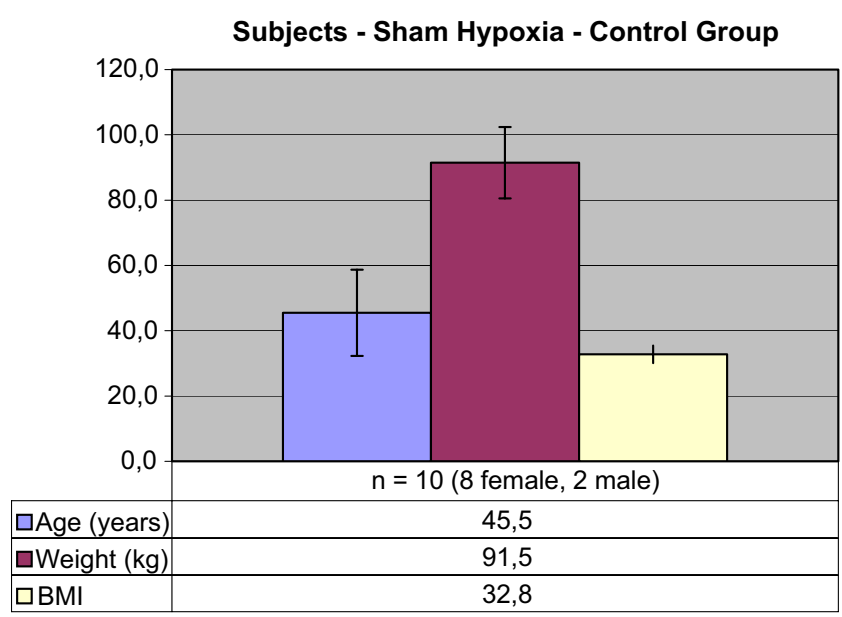

Fig. 3 Mean Age, weight, and BMI of the subjects who finished the trial in the sham hypoxia group 
Difference in Weight Reduction

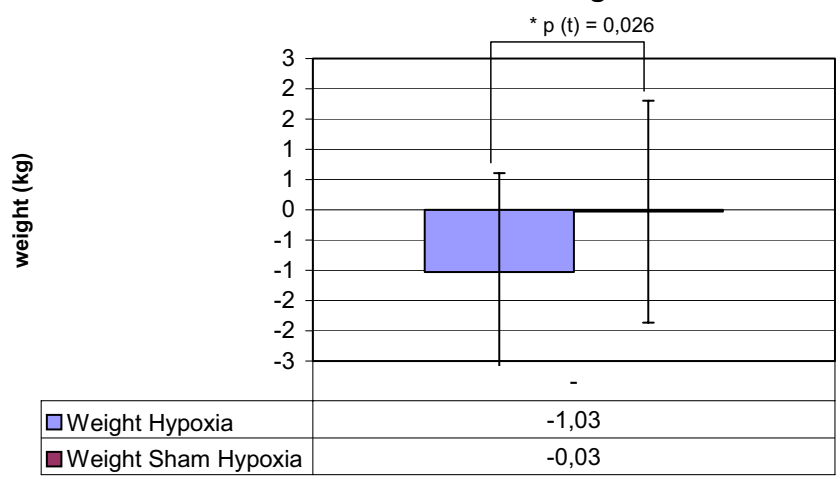

Fig. 4 Mean (SD) weight loss after 7 weeks in the hypoxia group vs the sham hypoxia group

Our aim was to perform a physical exercise weight loss study in obese subjects under moderate hypoxia (15 vol.\% $\mathrm{O}_{2}$ ) and to compare the effects on body weight and fat metabolism to a control group training on the same level in sham hypoxic conditions.

\section{Materials and methods}

Site Our facility for simulated altitude in the renovated Kurmittelhaus der Moderne in Bad Reichenhall in southeast Bavaria has three rooms $\left(90,37\right.$, and $\left.25 \mathrm{~m}^{2}\right)$ in which a hypoxic climate at normobaric conditions can be produced up to an simulated altitude of $6,000 \mathrm{~m}$ (approximately 7 vol. $\% \mathrm{O}_{2}$; see Fig. 1).

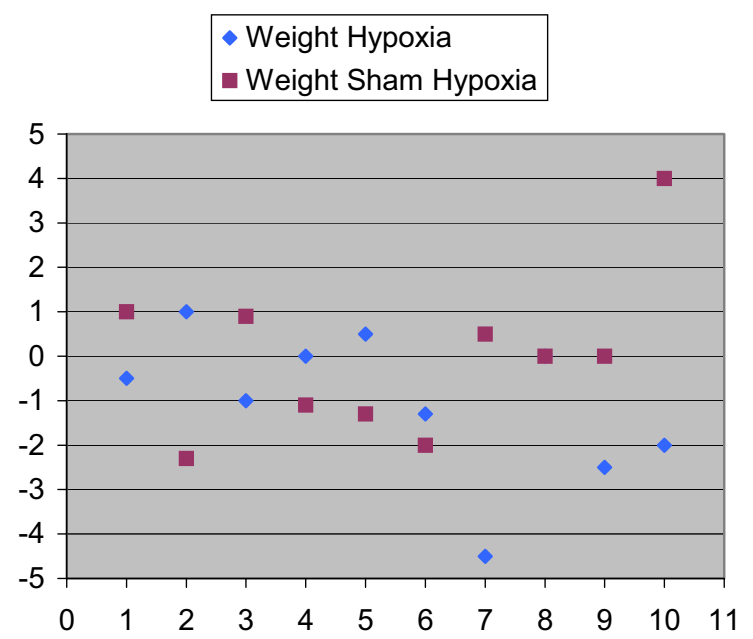

Fig. 5 Weight reduction or increase in the single subjects (matched pairs by age) of the hypoxia vs sham hypoxia group ( $y$-axis $=\mathrm{kg}, x$ axis $=$ number of subject). Weight loss occurred in six subjects of the hypoxia group and in four of the sham hypoxia group, weight increase in two of the hypoxia group and four of the sham hypoxia group, weight stayed unchanged in two subjects of hypoxia (four and eight) and two of the sham hypoxia group (eight and nine)
The rooms are two well-equipped training facilities and one sleeping and rest room with a full bath. To reach a hypoxic climate, the air in the room is flushed with nitrogen, and the volume percent of oxygen is reduced to the determined level. To control the climate, oxygen and carbon dioxide are constantly controlled. The data are transmitted to a computer, and the computer controls via specialized software the electronic climate unit and the inflow of nitrogen and fresh air through the air condition (software by Low Oxygen Systems, Berlin-Buch, Germany; hardware by Atlas Copco, Walter Bau and Siemens and Fujitsu-Siemens, all Germany). The nitrogen is produced via a filtration system that eliminates oxygen from pressurized air. Because of the constant air flow, there is a low noise level from the air condition in the room. The level of simulated altitude is usually displaced in the rooms. To keep subjects uninformed about the oxygen level in the room while the air condition was running at all times, this display was covered during the study times and made invisible to the subjects.

Subjects Thirty-two healthy subjects ( 22 men, 10 women) with a minimum age of 16 years and a minimum body mass index (BMI) of 27 were asked to participate in an 8-week trial with three times 90 -min moderate physical exercise. Estimation of sample size was calculated to reach a statistical significance for a change in BMI of one point. Acquisition of subjects was done by local radio announcement and personal contact. Subjects did not receive any kind of reimbursement for participation. All subjects were asked to continue with their normal diet/nutrition which they had before the study.

Twenty subjects were finally willing to perform the whole 8-week training period and gave written consent. Subjects were randomly allocated to the hypoxia training and the sham hypoxia training group. Subjects were not told to which group they were allocated. Antropometric data of the subjects in the two groups are shown in Figs. 2 and 3. Both groups were comparable in terms of age and sex.

In all 20 subjects, spiro-ergometry was performed to determine the max $\mathrm{O}_{2}$ consumption $\left(\mathrm{ml} \mathrm{kg}^{-1} \mathrm{~min}^{-1}\right)$ at exercise and the training level for the study set at the heart frequency value at $60 \%$ of the max $\mathrm{O}_{2}\left(\mathrm{ml} \mathrm{kg}^{-1} \mathrm{~min}^{-1}\right)$. In all subjects, an echocardiography was performed for safety reasons to exclude subjects with heart insufficiency levels NYHA 2-4.

Study parameters Primary study parameter was bodyweight $(\mathrm{kg})$ before and after the trial. Secondary study parameters were: BMI and the laboratory parameters (serum and EDTA blood sample); HbAC1, cholesterol, high- and low-density lipoproteins and triglycerides. Other exercise and blood parameters were not part of this trial but 
are available on request for reviewers from the laboratory and exercise test data sheets.

Blood samples were drawn at the beginning and end of the 8-week trial; bodyweight and height were measured via a regular medical weight scale.

Training period Subjects performed exercise at a heart rate level which corresponded to $60 \%$ of the heart rate level at their individual max $\mathrm{O}_{2}\left(\mathrm{ml} \mathrm{kg}^{-1} \mathrm{~min}^{-1}\right)$ on three different training (stepper, treadmill, and bicycle ergometer) devices for 90 min on 3 days per week, 8 weeks, either in normobaric hypoxia ( $15 \mathrm{vol} . \% \mathrm{O}_{2}$, equivalent to $2,500-\mathrm{m}$ altitude) or in normobaric normoxia $\left(20\right.$ vol. $\% \mathrm{O}_{2}$, equivalent to $450-\mathrm{m}$ altitude). For safety reasons, $\mathrm{SaO}_{2}$ was controlled regularly during the training via pulseoximeter (Konica Minolta, Japan) in all subjects, and the subjects were asked for the main symptoms of acute altitude sickness (headache, nausea, and training untypical weakness in the legs). Pulse oximetry was blinded to subjects. Training was under constant control of medical personnel.

Statistical analysis The differences between the two groups were analyzed via Student's $t$ test, statistical significance determined as $p<0.05$ (statistical software used: Excel, Microsoft, Redwood, WA, USA). Data are presented as descriptive data, and values are given as mean values.

Ethics and informed consent The study protocol was approved by the ethical committee of the Paris Lodron University Salzburg. All subjects gave written informed consent to participate in the study. Subjects under 18 were finally excluded; therefore, written consent by the parents was not necessary.

\section{Results}

All 20 subjects who were included in the training sessions finished the study without problems. No adverse side effects were reported by the subjects, including no signs of acute mountain sickness in those who exercised in 15.2 vol.\% oxygen level.

The average heart rate at $60 \% \mathrm{O}_{2}$ max for all subjects was $118 / \mathrm{min}$. Subjects kept this heart frequency level stable during exercise $( \pm 3 / \mathrm{min})$. This meant that because of the slightly increased heart frequency at rest in hypoxia, the hypoxia group exercised at a lower wattage.

Because of the neutral smell of the air-conditioned air, subjects could not tell if they are in hypoxia or sham hypoxia. More than $50 \%$ of the subjects guessed wrongly as to which group they belong.

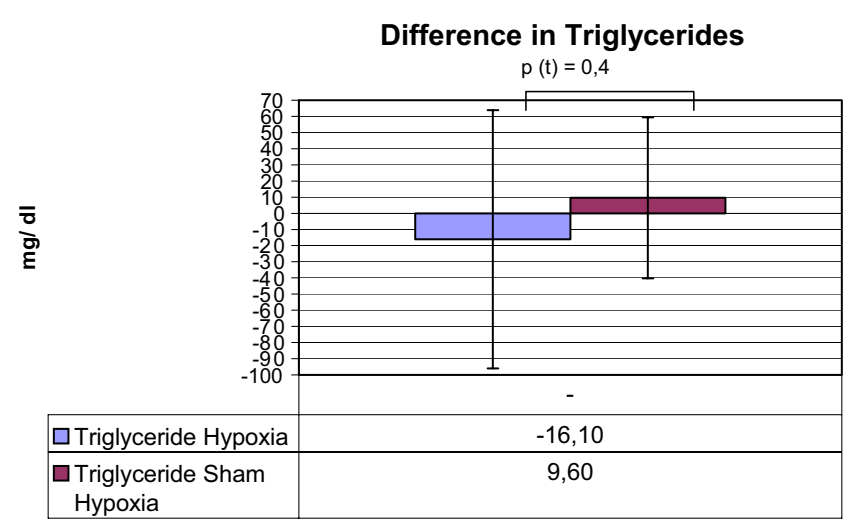

Fig. 6 Mean (SD) reduction and increase in triglycerides values of the hypoxia vs sham hypoxia group

Weight loss: The hypoxia group lost $1.14 \mathrm{~kg}$ in mean; the sham hypoxia group lost no bodyweight at all ( $p=0.02$, see Figs. 4 and 5). This led to a non-statistical significant tendency in the hypoxia group to reduce the BMI more than the sham hypoxia group $(p=0.33)$. The number of subjects had been too low to reach statistical significance.

Lipid levels and HbA1C: None of the differences in lipid levels reached statistical significance. However, the hypoxia group showed little non-statistical significant tendencies to lose more cholesterol $(-10.7 \mathrm{mg} / \mathrm{ml} \mathrm{vs} 6.0 \mathrm{mg} / \mathrm{ml} ; p=0.68)$, triglycerides, and low-density lipoproteins than the sham hypoxia group, but less high-density lipoproteins (Figs. 6 and 7).

The healthy subjects of both groups were not diabetics. They had HbA1C levels on the edge but still in normal values (mean 5.67 and 5.47\%), and those levels did not change after the training period of 8 weeks.

\section{Discussion}

The here presented study on fat metabolism and weight loss in mild to moderate hypoxia is, to our knowledge, the first one with a sham hypoxia control protocol.

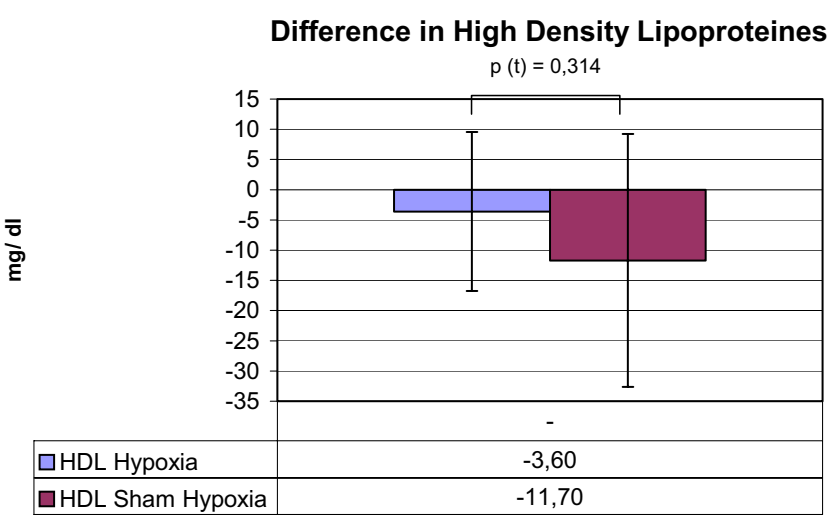

Fig. 7 Mean (SD) reduction in high-density lipoproteins $(H D L)$ in the hypoxia vs sham hypoxia group 
We could show that obese subjects did lose significantly more weight during an 8-week training period with three times per week 90-min low intense exercise when this exercise took place in 15 vol. $\% \mathrm{O}_{2}$ hypoxia than in normal air at $450-\mathrm{m}$ altitude $\left(20\right.$ vol.\% $\left.\mathrm{O}_{2}\right)$. We could also show that there have been non-statistical differences in fat metabolism or at least in serum levels of lipoproteins and triglycerides between the hypoxia training and the sham hypoxia training group.

There are, due to the nature of the study and above that, several limitations, which have to be addressed. The number of subjects is not big enough to show a reduction in BMI, and power analysis shows that we would need about 32 subjects to have a significant BMI reduction. We wanted to get more subjects into the study, but the reduction of subjects to a limited number due to time restrictions and willingness is a common problem in weight loss protocols with obese people. The difference in weight loss in mean with $1.14 \mathrm{~kg}$ seems, for 8 weeks of training, not really a lot. However, several subjects did significantly lose more weight in the hypoxia group, and the mean bodyweight loss is not due to natural swings in weight change.

This also happened although the hypoxia group trained at a slightly lower wattage due to a slightly increased heart frequency in hypoxia. We cannot fully exclude that the normoxia group decreased body fat and increased muscle mass due to the slightly higher level of muscle load, but it seems unlikely with this very low intense training.

Because we did not put our subjects on a stringent diet, there might have been the chance that some individuals did eat more than normally and some less. Keeping the subjects on their routine food seemed to us the natural choice of being uninfluenced in the weight change by a special nutritional diet. The other point is that weight loss in hypoxia might be caused by reduced food intake, and that was a part of the study. To let the subjects reduce their food intake, you have to have them on a longer leash. This is especially true if leptin and hypothalamic signals come into play and might let subjects be less hungry, respectively, less hungry for fat. To achieve more accuracy, the only chance would be to exactly control the nutrition of all subjects already before the study and during the training period. To do this, subjects have to be under full supervision all the time. This is an almost impossible achievement in ambulatory care or with independent volunteers.

Our findings are consistent with previous studies which have shown that low intense training in moderate hypoxia leads to weight loss [11-14]. The protocol with a sham hypoxia control group, which did not lose weight with the same amount of exercise, strengthens this hypothesis. A definite reason why this weight loss occurs is still not known. Several investigations could prove that the body reduces fat as a substrate for energy production due to the higher use of oxygen necessary in fat burning [2, 13], but this is still controversially discussed [14]. One of the reasons for a controversial discussion is that so far, nobody did observe subjects on a longer term [14]. Most studies have dealt with exercise in hypoxia for hours or a maximum of a few days, not for 2 months as we did.

In our investigation, the subjects in hypoxia had reduced their triglycerides, their cholesterol, and their low-density lipoproteins and kept their high-density lipoproteins stable, whereas the subjects in normoxia did not do so. However, none of the effects reached statistical significance. Due to the actual literature, this can be explained by several possible causes: Either the fat mobilization is reduced due to hypoxia over time and so the serum levels of lipids are lower at the point of measurement or the increased catecholamine levels during hypoxia lead to reduced lipolysis and a depot of intracellular fat $[16,17]$.

Most likely, it seems that increased leptin levels lead to a reduced fat intake via food, and that is the reason why triglyceride levels decrease $[6,10]$. The answer is still open, and more research is needed to clarify this. Other mediators besides catecholamines, like insulin like growth factor, might influence both fat and carbohydrate metabolism in hypoxia [15].

In conclusion, there is more and more evidence that even mild moderate hypoxia in comfortable simulated altitude, if combined with exercise, leads to weight loss.

Weight loss regimes with exercise and diets or just exercise could be combined with normobaric hypoxia in the future. Future investigations should include whether the stay in moderate hypoxia alone without exercise increases the weight loss and if such a regimen would still be safe.

\section{References}

1. Hackett PH, Roach HC (2001) High-altitude illness. N Engl J Med 345:1279

2. Roberts AC, Butterfield G, Cymerman A, Reeves J, Wolfel E (1996) Acclimatization to 4.300-m altitude decreases reliance on fat as a substrate. J Appl Physiol 8(4):1762-1771

3. Losbaugh JE, Loeppky JA, Greene ER (2006) Effects of acute hypobaric hypoxia on resting and postprandial 1 superior mesenteric artery blood flow. High Alt Med Biol 7:47-53

4. Westerp KR (2001) Energy and water balance at high altitude. News Physiol Sci 16:134-137

5. Kayser B (1992) Nutrition and high altitude exposure. Int J Sports Med 13(Suppl 1):129-132

6. Tschopp M, Strasburger CJ, Hartman G et al (1998) Raised leptin levels at high altitude associated with loss of appetite. Lancet 312:1119-1120

7. Guerre-Millo M, Grosfeld A, Issad T (2002) Leptin is a hypoxia inducible gene. Obes Res 10:857-858

8. Simler N, Grosfeld A, Peinnequin A et al (2006) Leptin receptor deficient Zucker rats reduce their food intake in response to hypobaric hypoxia. Am J Physiol Endocrinol Metab 290:E591-E597 
9. Grosfeld A, Zilberfarb V, Turban S et al (2002) Hypoxia increases leptin expression in human PAZ6 adipose cells. Acta Diabetol 45:527-530

10. Yingzhong Y, Droma Y, Rili G, Kubo K (2006) Regulation of body weight by leptin, with special reference to hypoxia induced regulation. Intern Med 45:941-946

11. Isbary GH (2006) Einfluss von normobarer Hypoxie auf die Fettutilisation bei einer Laufausdauerbelastung. Dissertation zur Erlangung des Doktorgrades der Medizin, Universität Tübingen

12. Jones N, Robertson D, Kane J, Hart RA (1982) Effect of hypoxia on free fatty acid metabolism during exercise. J Appl Physiol 33 (6):733-738

13. Boyer J, Blume FD (1984) Weight loss and changes in body composition at high altitude. J Appl Physiol 57:1580-1585
14. Schobersberger W, Schmid P, Lechleitner M et al (2003) Austrian Moderate Altitude Study 2000 (AMAS 2000). The effects of moderate altitude $(1700 \mathrm{~m})$ on cardiovascular and metabolic variables in patients with metabolic syndrome. Eur J Appl Physiol $88: 506-514$

15. Schmidt W, Dore S, Helgendorf A, Strauch S (1995) Effects of exercise during normoxia and hypoxia on the growth hormoneinsulin-like growth factor I axis. Eur J Appl Physiol 71(5):424-430

16. Strobel G, Neureither M, Bärtsch P (1996) Effect of acute mild hypoxia during exercise on plasma free and sulphoconjugated catecholamines. Eur J Appl Physiol 73(1-2):82-87

17. Richalet JP, Mehidioi H, Rathat C, Vignon P, Keromes A (1998) Acute hypoxia decreases cardiac response to catecholamines in exercising humans. Int J Sports Med 9:157-162 